\title{
Roberta IOLI (ed.), Gorgia. Testimonianze e frammenti. Introduzione, traduzione e commento
}

\author{
Stefania Giombini
}

\section{(2) OpenEdition}

\section{Journals}

Edizione digitale

URL: https://journals.openedition.org/philosant/831

DOI: $10.4000 /$ philosant.831

ISSN: 2648-2789

\section{Editore}

Éditions Vrin

\section{Edizione cartacea}

Data di pubblicazione: 1 novembre 2014

Paginazione: 325-328

ISBN: 978-2-7574-0855-1

ISSN: 1634-4561

\section{Notizia bibliografica digitale}

Stefania Giombini, «Roberta ıьı (ed.), Gorgia. Testimonianze e frammenti. Introduzione, traduzione e commento », Philosophie antique [En ligne], 14 | 2014, mis en ligne le 01 novembre 2018, consulté le 02 décembre 2022. URL : http://journals.openedition.org/philosant/831 ; DOI : https://doi.org/10.4000/ philosant.831

\section{(c)}

Creative Commons - Attribution - Pas d'Utilisation Commerciale - Pas de Modification 4.0 International - CC BY-NC-ND 4.0

https://creativecommons.org/licenses/by-nc-nd/4.0/ 
s'échanger indéfiniment » (p. 133). À défaut d'une explication précise de ce rôle de liant, une analogie est tracée avec l'élégie de Tyrtée, dans laquelle l'invocation de « tout homme quel qu'il soit » permet d'obtenir par avance l'accord de tout membre de la communauté des hommes. Mais comment l'invocation du verbe être pourrait-elle produire le même effet d'inclusion dans une communauté ? Nous devons à cet égard nous contenter de l'affirmation que le verbe être est la condition d'un « rapport simultané d'identité et de différence » entre le je et le $t u$, de sorte qu' « à leur origine semble encore, toujours et déjà se trouver l'essence linguistique du verbe être » (p. 135). On ne peut s'empêcher tout de même de se demander si ce montage linguistique n'est pas sensiblement plus anachronique que l'attribution à Parménide d'une réflexion sur la signification de « être ».

Pour jouer à notre tour avec les bouclages de boucles, nous conclurons que le lecteur du présent ouvrage qui s'estimerait insatisfait du manque de persuasion de l'argumentation n'aurait décidément rien compris à Parménide.

Annick STEVENS

Roberta IOLI (ed.), Gorgia. Testimonianze e frammenti. Introduzione, traduzione e commento, Rome, Carocci editore, 2013, 327 pages, ISBN 978-88-430-6892-0

Da alcuni anni si registra una crescita di interesse per Gorgia di Leontini e le sue opere, testimoniata dal buon numero di studi e pubblicazioni che con una certa continuità si offrono sia nell'ambito della filosofia che della cultura classica in generale; questo è senz'altro di buon auspicio per un avanzamento della comprensione del pensiero di questo intellettuale siceliota.

Il panorama italiano non è estraneo a questa tendenza, anzi si può dire che esso presenti un quadro ricchissimo di ricerche e studi di spessore: già solo guardando alle ultime due decadi si sono affermati numerosi studi nella letteratura critica anche internazionale ${ }^{1}$. Ora, a queste opere si aggiunge il volume a cura di Roberta Ioli per l'Editore Carocci nella collana Classici. Ioli, che aveva già presentato una traduzione del Peri tou me ontos di Gorgia nel 2010, in questo nuovo lavoro propone tutti i frammenti e le testimonianze gorgiane apportando alcune significative varianti rispetto all'edizione Diels-Kranz di suo riferimento (come esplicitato in chiusura nella «Nota al testo», p. 47).

Il lavoro è aperto da un ampio saggio introduttivo, «Gorgia e i "vortici della lingua” $\gg$, che intende mostrare la prospettiva ermeneutica della studiosa e che offre un'ampia analisi delle questioni che ruotano intorno al sofista. Ioli prende posizione nella disputa sulla figura di Gorgia che si è riproposta in maniera pressoché costante nel dibattito storiografico, incentrata sulla questione se Gorgia sia un filosofo o esclusivamente un retore, e sostiene che Gorgia è un filosofo-retore, in

1. Vd. L. Càffaro, Gorgia. Encomio di Elena, Apologia di Palamede, Firenze, Aletheia, 1997; G. Mazzara, Gorgia, la retorica del verosimile, Sankt Augustin, Academia Verlag, 1999; G. Paduano, Gorgia. Encomio di Elena, Napoli, Liguori, 2004; S. Mariani, In difesa di Palamede, Genova, Il Melangolo, 2010; S. Giombini, Gorgia epidittico. Commento filosofico all'Encomio di Elena, all'Apologia di Palamede, all' Epitaffio, Passignano s.T., Aguaplano, 2012; per le edizioni delle testimonianze e dei frammenti de I Sofisti cf. l'edizione di M. Bonazzi nella collana Bur, Milano 2007, e la riedizione di M. Untersteiner e A. M. Battegazzore, Milano, Bompiani, 2009. 
grado di incarnare contemporaneamente le due figure, apparentemente in opposizione. Partendo dall'analisi del Peri tou me ontos (ptmo), l'idea centrale dello studio è che questo sia un 'opera filosofica' in cui la modalità dell'argomentazione di natura dialettica si presta all'analisi del linguaggio e del rapporto tra realtà e conoscenza (p. 12). Proseguendo l'analisi delle altre opere gorgiane, Ioli usa in maniera predominante il termine «dialettico» in quanto esso sarebbe il filo conduttore di tutta la produzione del sofista, la cifra della sua metodologia speculativa. Proprio la struttura dialettica rende il ptmo e gli altri logoi gorgiani ben più che semplici antilogie. Infatti, mentre un'antilogia si limita ad essere «un'antitesi puntuale delle argomentazioni dell'avversario» (p. 12) senza dunque assurgere a vera e propria argomentazione logica, invece un logos dialetticamente elaborato esprime la crescita della conoscenza propria della filosofia, che tende continuamente verso la verità senza mai poterla fissare. In tal senso, Gorgia sembra approssimarsi alla strategia diafonica che in seguito sarà tipica dei pirroniani: Ioli preferisce collegare Gorgia allo scetticismo sebbene dal suo punto di vista egli non risulti avvicinabile a questo orientamento di pensiero (p. 24), e poi delinea, forse velocemente, un Gorgia relativista il cui logos paradossale da un lato non può fondare niente, dall'altro può creare la realtà raccontandola in molteplici modi. Le opere di Gorgia non sono neanche ascrivibili, per la studiosa, al solo genere epidittico, dal momento che tale definizione non pare sufficiente ad esplicitarne la natura: il termine risulta essere piuttosto vacuo. Quest'ultima valutazione è parzialmente riesaminata quando Ioli rileva che le opere di Gorgia sono a pieno titolo epidittiche in quanto didatticamente fertili (p. 28).

Il ptmo supera la riflessione ontologica proprio perché il linguaggio paradossale mostra l'inammissibilità di una sola verità. Dunque il trattato gorgiano intende esibire la debolezza delle ontologie eleatiche e l'impossibilità di raggiungere una unica verità, un valore assoluto. Così, tutta la produzione del sofista indaga e presenta lo smacco per l'inaccessibilità alla verità e per questo giunge ad esaltare il valore poietico della parola: la parola che fa, che crea la realtà. Gorgia ne esce, alfine, come una sorta di tecnico-scienziato della parola che raggiunge un esito poetico (p. 17). La parola, dunque, produce una realtà ingannando, non potendo essa portare verità, ma questo inganno non solo è esteticamente affascinante, esso è anche eticamente sostenibile in quanto unica possibile via della ricerca filosofica: è qui che, per Ioli, il retore e il filosofo vengono a coincidere.

Nella successiva «Nota al testo» Ioli propone degli utili apparati concernenti la tradizione manoscritta e riflette sia sulla cronologia, sostenendo l'idea che «nel caso del Palamede, verosimilmente posteriore al ptmo e anteriore all'Elena, la datazione dovrebbe essere prossima al trattato» [440-430] (p. 41) sia sull'identità dell' Anonimo De $M X G$, convenendo che sia autore peripatetico.

Dopo le premesse teoretiche e tecniche Ioli presenta le testimonianze, i frammenti, le imitazioni e gli addenda. Per ogni sezione è fornita la stessa organizzazione testuale: originale greco con a fronte la traduzione italiana e in coda il commento per ogni singolo testo. Come detto, Ioli utilizza l'edizione DK ma ha cura di ampliare quando ritiene che un'estrapolazione più corposa possa garantire una maggiore comprensione: è il caso di B23 (Plut. De glor. Ath. 5, p. $348 \mathrm{C}$ ) che ne guadagna in informatività, o il caso di B2 (Olymp., In Plat. Gorg. 9.1-9) che rife- 
risce del rapporto tra Gorgia e Platone («la testimonianza di Olimpiodoro ci conferma l'impegno filosofico, e non solo retorico, di Gorgia», p. 156). Quanto alle integrazioni più importanti si deve registrare l'inserimento della versione dell' Anonimo De $M X G$ in B3a. L'originario frammento DK contenente la versione di Sesto viene denominato $3 \mathrm{~b}$ (nell'edizione Giannantoni già 3a; http://ancient source.daphnet.org/). Ioli motiva l'inserimento e soprattutto la precedenza di $3 \mathrm{a}$ rispetto a $3 \mathrm{~b}$ sostenendo che la versione dell'Anonimo è ben più attendibile di quella di Sesto: la versione di Sesto, infatti, non è dimostrativa dell'argomento proprio di Gorgia (una specifica modalità argomentativo-dimostrativa di Gorgia ${ }^{1}$ che consiste nel giocare con la ricchezza semantica di einai al fine di attaccare la dottrina eleatica, p. 160) poiché si risolve tramite l'eliminazione ad uno ad uno dei corni del trilemma ontologico (p. 184).

Nella sezione Addenda Ioli aggiunge, a ragione, la fonte plutarchea De gen. Socr. 538 B relativa al ritorno definitivo di Gorgia in patria, richiamando in tal modo il lavoro di R. Velardi: Gorgia a Tebe, l'incontro con il pitagorico Liside e il ritorno definitivo in Sicilia: Plut. De gen. Socr. 583 B. Una testimonianza non inclusa nei Fragmente der Vorsokratiker, «AION» 20, 1998, 89-106 (p.39). Nell'ottica di una maggiore completezza, e per pertinenza, sarebbe stato bene includere anche un'altra testimonianza: Plut. De aud. poet. 1 p. $15 \mathrm{C}$, che non è presente in DK ed è invece inclusa in Untersteiner (B23a: «Gorgia a chi aveva chiesto "Perché solo i Tessali non inganni?", rispose: "Perché sono troppo privi di un' assennata esperienza per venir ingannati da me" ») e accolta nell'edizione Giannantoni (B23aG). Questo frammento si integrerebbe bene col fr. B23.

Roberta Ioli conferma una validissima traduzione, fluente, piacevole, curata nei particolari, anche con piccole pregevoli note, come in A24 dove rivede la tradizionale traduzione di narthex intendendolo nel senso non di ferula ma di verga, termine che fa risaltare meglio la forza polemica di Gorgia.

Riguardo alla sezione dei commenti, nella prima parte dedicata alle testimonianze, la studiosa ha cura di inquadrare la fonte, il suo contesto e il contenuto senza mai dimenticare il confronto con la letteratura critica; nella seconda parte dedicata ai frammenti il commento si arricchisce ulteriormente con l'analisi dello stile, delle eventuali ricostruzioni dei manoscritti, delle parti del discorso e delle analisi lessicali. Di particolare interesse alcune analisi dei termini del campo semantico come, ad esempio, quello inerente la colpa con hamartema, adikema, atychema e aitia, che dimostrerebbe non solo la grande abilità lessicale di Gorgia ma anche che «ben prima di Aristotele, Gorgia sviluppa il vocabolario della colpa» (p. 232).

Da sottolineare che le sezioni dei commenti contengono un'amplissima quantità di riferimenti, connessioni concettuali, richiami tra autori e soprattutto rimandi di natura bibliografica. I commenti sono senza dubbio una sezione pregevole, la più efficace del volume, oltre alla traduzione, perché sono uno strumento di lavoro ad ampio raggio, dotato di un fortissimo grado di esaustività. Vorremmo solo segnalare il rischio che blocchi tanto corposi di informazioni possano non mettere in luce argomenti e tematiche a cui potrebbe valere la pena dare maggiore

1. Cf. M. Wesoly, La «Dimostrazione propria» di Gorgia, «Peitho/Examina Antiqua» 1 (4) 2013, p. 159-188. 
risalto. In tal senso, si nota la mancanza di un index nominum et locorum; un volume così ricco, anche in richiami bibliografici, non può farne a meno, pena una diminuita fruibilità: è auspicabile una integrazione in seconda edizione. C'è, inoltre, la possibilità che, a volte, $\mathrm{i}$ commenti siano più concentrati sull'analisi e meno sulla ricostruzione di una visione di insieme. Solo a mo' di esempio: Ioli riconosce al par. 7 dell'Elena la distinzione tra zemia e atimia, ne nota l'inapplicabilità per un barbaro quale era Paride, ma non lo inquadra nel contesto della possibile ricostruzione di un sapere giuridico in Gorgia: avrebbe potuto connetterlo al par. 1 dell'Elena dove si parla di responsabilità per la costrizione attraverso l'uso dei logoi o al par. 35 del Palamede dove si fa riferimento alla necessità della pena preventiva.

Quanto poi alla proposta interpretativa nel suo complesso, Ioli si focalizza sulla dimensione filosofica del sofista e si muove sostanzialmente su quelle rotte interpretative già battute nel corso del Novecento che vedono nel sofista un filosofo, un relativista, un artista della parola esteticamente potente: il ritratto di Gorgia che ne deriva si allinea all'idea già codificata dalla storiografia.

In conclusione, il volume di Roberta Ioli si presenta come una raccolta pregevolissima delle testimonianze e dei frammenti gorgiani in lingua italiana: crediamo che il volume si imporrà, e meritatamente, come strumento di riferimento per tutti gli studiosi e gli estimatori di Gorgia.

Stefania GIOMBINI

Catalin Partenie (éd.), Plato's Myths, Cambridge, Cambridge University Press, 2009, 255 pages, ISBN 978-0-521-88790-8.

Ce volume rassemble une dizaine d'études inédites, précédées d'une préface générale, portant sur l'articulation entre mythe et philosophie dans l'œuvre de Platon. Plus spécifiquement, la question qui intéresse les études ici réunies est celle du rapport complexe entre les récits mythiques que l'on voit apparaître dans les dialogues et le projet proprement philosophique (dialectique) de Platon. Cette question est d'autant plus prégnante que Platon lui-même ne cesse de problématiser la fonction des mythes au sein de l'œuvre. Comment, en effet, légitimer le recours à une forme d'expression que l'on récuse explicitement en vertu de son incompatibilité méthodologique avec les prétentions à la vérité de la dialectique ? La question peut se poser aussi pour d'autres formes d'expression ou de raisonnement, tels l'exemple, l'analogie et le paradigme, que Platon emploie à dessein en dépit des tensions méthodologiques qu'elles créent. Or, comme le montre l'ensemble des contributions, ce qui rend le cas du mythe platonicien si attrayant pour l'historien de la philosophie ancienne est que sa légitimation s'appuie sur des notions qui sont elles-mêmes philosophiquement problématiques, telles que la vérité (aletheia), la persuasion (pistis) et le vraisemblable (eikos).

Ces dernières années, l'articulation entre mythe et philosophie dans l'œuvre de Platon a été cernée à partir de deux positions interprétatives opposées. La première, d'inspiration ontologique, cherche à effacer la tension existante entre le discours proprement dialectique et les mythes. Dans cette perspective, le recours au mythe, tout comme le recours à la forme dialogique, témoignerait d'un mouvement fictionnel inhérent au langage qui, en visant le locus d'une vérité insaisissable, 